\title{
SIGNLESS AND NORMALIZED LAPLACIAN SPECTRUMS OF THE POWER GRAPH AND ITS SUPERGRAPHS OF CERTAIN FINITE GROUPS
}

\author{
A. HAMZEH \\ Department of Pure Mathematics \\ Faculty of Mathematical Sciences, University of Kashan \\ Kashan 87317-53153, I. R. Iran
}

\begin{abstract}
The aim of this article is to compute the signless and normalized Laplacian spectrums of the power graph, its main supergraph and cyclic graph of dihedral and dicyclic groups.

Key words and Phrases: Power graph, signless Laplacian, normalized Laplacian, cyclic graph, main supergraph.
\end{abstract}

\begin{abstract}
Abstrak. Tujuan dari paper ini adalah untuk menghitung spektrum Laplasian tanpa tanda dan dinormalkan dari graf pangkat, super graf utamanya, dan graf siklis dari grup disiklis dan dihedral.

Kata kunci: Graf pangkat, Laplasian tanpa tanda, Laplasian dinormalkan, graf siklis, supergraf utama.
\end{abstract}

\section{Basic Concepts}

All groups in this paper are assumed to be finite and graphs are simple. Let $\Gamma$ be a graph with vertex set $V(\Gamma)$ and edge set $E(\Gamma)$. For the vertex $i$ in $V(\Gamma), d_{\Gamma}(i)$ is degree of $i$ that is equal to the number of neighbors of $i$. If each vertex of graph, has the same degree of $r$, then graph is $r$-regular. The adjacency matrix $A(\Gamma)$, Laplacian matrix $L(\Gamma)$, signless Laplacian matrix $Q(\Gamma)$ and normalized Laplacian

2000 Mathematics Subject Classification: 05C25, 05 C50.

Received: 04-04-2017, revised: 10-06-2017, accepted: 11-06-2017. 
matrix $\mathcal{L}(\Gamma)$ are defined as follows:

$$
\begin{aligned}
A(\Gamma)=\left(a_{i j}\right) & =\left\{\begin{array}{cc}
1, & i j \in E(\Gamma) \\
0, & \text { otherwise }
\end{array}\right. \\
L(\Gamma) & =D(\Gamma)-A(\Gamma), \\
Q(\Gamma) & =D(\Gamma)+A(\Gamma), \\
\mathcal{L}(\Gamma)=\left(\mathcal{L}_{i j}\right) & = \begin{cases}1, & i=j \text { and } d_{\Gamma}(i) \neq 0 \\
-\frac{1}{\sqrt{d_{\Gamma}(i) d_{\Gamma}(j)}}, & i j \in E(\Gamma) \\
0 & \text { otherwise }\end{cases}
\end{aligned}
$$

where $D(\Gamma)$ is the diagonal matrix of degrees. If $N$ is a square matrix, then $P(N, x)=\operatorname{det}(x I-N)$ is characteristic polynomial of $N$. The multi-sets of all eigenvalues, Laplacian eigenvalues, signless Laplacian eigenvalues and normalized Laplacian eigenvalues of $\Gamma$ are denoted by $\operatorname{Spec}(\Gamma), \operatorname{Spec}_{L}(\Gamma), \operatorname{Spec}_{Q}(\Gamma)$ and $\operatorname{Spec}_{\mathcal{L}}(\Gamma)$, respectively. We usually write $\operatorname{Spec}(\Gamma)=\left\{\lambda_{1}^{\left(s_{1}\right)}, \ldots, \lambda_{m}^{\left(s_{m}\right)}\right\}$, where $\lambda_{1}, \ldots, \lambda_{m}$ are distinct $\Gamma$-eigenvalues and $s_{j}$ is the multiplicity of $\lambda_{j}, 1 \leq j \leq m$.

Following Sabidussi [17, p. 396] the $A$-join of a set of graphs $\left\{\Gamma_{a}\right\}_{a \in A}$ is the graph $\Delta$ with the following vertex and edge sets:

$$
\begin{aligned}
& V(\Delta)=\left\{(x, y) \mid x \in V(A) \& y \in V\left(\Gamma_{x}\right)\right\} \\
& E(\Delta)=\left\{(x, y)\left(x^{\prime}, y^{\prime}\right) \mid x x^{\prime} \in E(A) \text { or else } x=x^{\prime} \& y y^{\prime} \in E\left(\Gamma_{x}\right)\right\} .
\end{aligned}
$$

For the $p$-vertex graph $A$, the $A$-join of $H_{1}, H_{2}, \cdots, H_{p}$ is denoted by $A\left[H_{1}, H_{2}, \cdots, H_{p}\right]$.

Let $G$ be a finite group. For $x \in G$, the order of $x$ is denoted by $o(x)$. The set of all element orders of $G$ is denoted by $\pi_{e}(G)$ and the number of all elements of $G$ of order $i$ is denoted by $\Omega_{i}(G)$. The investigation of graphs related to algebraic structures is important, because graphs like these have important applications (see, for example, [10]) and are related to automata theory (see [8]). The Cayley graph is the oldest simple graphs associated to the finite group $G$. The power graph was introduced by Kelarev and Quinn in [13]. Two elements $x, y \in G$ are adjacent in the power graph if and only if one is a power of the other. Cameron and Ghosh in [1], proved that abelian groups with the same number of elements of each possible order can be characterized by their power graphs. For more information on power graphs see $[2,9,11,12]$. The cyclic graph $\Gamma_{G}$ is a simple graph with the vertex set $G$. Two elements $x, y \in G$ are adjacent in the cyclic graph if and only if $\langle x, y\rangle$ is cyclic [14]. Set $\pi_{e}(G)=\left\{a_{1}, \ldots, a_{k}\right\}$ and define the graph $\Delta_{G}$ with vertex set $\pi_{e}(G)$ and edge set $E\left(\Delta_{G}\right)=\left\{x y\left|x, y \in \pi_{e}(G), x\right| y\right.$ or $\left.y \mid x\right\}$. In $[4,5]$, the present author introduced the main supergraph $\mathcal{S}(G)$, that is a graph with vertex set $G$ in which two vertices $x$ and $y$ are adjacent if and only if $o(x) \mid o(y)$ or $o(y) \mid o(x)$. In the mentioned papers, they proved that $\mathcal{S}(G)=\Delta_{G}\left[K_{\Omega_{a_{1}}(G)}, \ldots, K_{\Omega_{a_{k}}(G)}\right]$, where $K_{n}$ denotes the complete graph on $n$ vertices. Note that the graphs $\mathcal{S}(G)$ and $\Gamma_{G}$ are supergraphs of the power graph. Laplacian spectrum of power graphs of 
finite cyclic and dihedral groups computed by Chattopadhyaya and Panigrahi in [3] and in [15], Mehranian et al. obtained spectrum of the power graphs of $D_{2 n}$, $T_{4 n}$ and $S D_{8 n}$. Also in $[6,7]$ the eigenvalues and Laplacian eigenvalues of $\mathcal{S}(G)$ are computed. Throughout this paper we refer to [16] for group theory concepts and for graph theoretical concepts and notations, we refer to [19].

In this paper we compute signless Laplacian and normalized Laplacian eigenvalues of the power graph and some of its supergraphs. As an application, signless Laplacian and normalized Laplacian eigenvalues of the power graph and its supergraphs are computed for the dihedral and dicyclic groups.

\section{Main Results}

The dihedral and dicyclic groups can be presented as follows:

$$
\begin{aligned}
D_{2 n} & =<a, b \mid a^{n}=b^{2}=1, b a b=a^{-1}>, \\
T_{4 n} & =<a, b \mid a^{2 n}=1, a^{n}=b^{2}, b^{-1} a b=a^{-1}>.
\end{aligned}
$$

We now state a result of [20] which is important in our next results.

Theorem 2.1. [20] Let $H$ be a graph with $V(H)=\{1,2, \ldots, k\}$, and $G_{i}$ 's be $r_{i}$ regular graphs of order $n_{i}(i=1,2, \ldots, k)$. If $\Gamma=H\left[G_{1}, G_{2}, \cdots, G_{k}\right]$, then signless Laplacian spectrum can be computed as follows:

$$
\operatorname{Spec}_{Q}(\Gamma)=\left(\bigcup_{i=1}^{k}\left(N_{i}+\left(\operatorname{Spec}_{Q}\left(G_{i}\right) \backslash\left\{2 r_{i}\right\}\right)\right)\right) \bigcup \operatorname{Spec}\left(\left(C_{Q}(H)\right),\right.
$$

where

$$
N_{i}= \begin{cases}\sum_{j \in N_{H}(i)} n_{j}, & N_{H}(i) \neq \emptyset \\ 0, & \text { otherwise }\end{cases}
$$

and

$$
C_{Q}(H)=\left(c_{i j}\right)_{k \times k}= \begin{cases}2 r_{i}+N_{i}, & i=j, \\ \sqrt{n_{i} n_{j}}, & i j \in E(H), \\ 0 & \text { otherwise. }\end{cases}
$$

The following result is an immediate consequence of Theorem 2.1 and the fact that $\operatorname{Spec}_{Q}\left(K_{n}\right)=\left\{2 n-2, n-2^{(n-1)}\right\}$.

Corollary 2.2. Suppose $\mathcal{S}(G)=\Delta_{G}\left[K_{\Omega_{a_{1}}(G)}, \ldots, K_{\Omega_{a_{k}}(G)}\right]$. Then the signless Laplacian spectrum of the main supergraph is computed as follows:

$$
\operatorname{Spec}_{Q}(\mathcal{S}(G))=\left(\bigcup_{i=1}^{k}\left(N_{i}+\Omega_{a_{i}}(G)-2\right)^{\left(\Omega_{a_{i}}(G)-1\right)}\right) \bigcup \operatorname{Spec}(C),
$$


where

$$
\begin{aligned}
N_{i} & = \begin{cases}\sum_{a_{j} \in N_{\Delta_{G}}\left(a_{i}\right)} \Omega_{a_{j}}(G), & N_{\Delta_{G}}\left(a_{i}\right) \neq \emptyset \\
0, & \text { otherwise }\end{cases} \\
\rho_{l, s} & =\rho_{s, l}=\left\{\begin{array}{ll}
\sqrt{\Omega_{a_{l}}(G) \Omega_{a_{s}}(G)}, & a_{l} \mid a_{s} \text { or } a_{s} \mid a_{l} \\
0, & \text { otherwise }
\end{array},\right.
\end{aligned}
$$

and

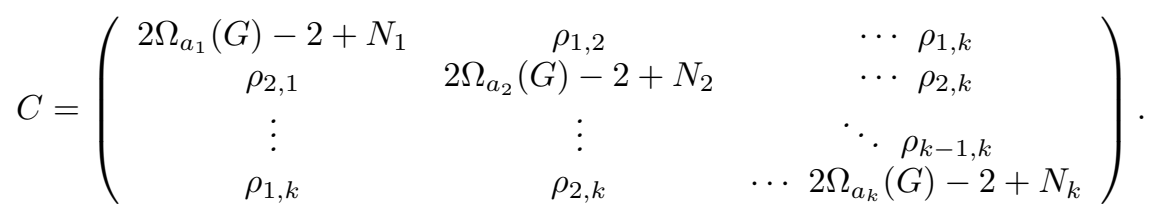

In the next results, the signless Laplacian spectrum of the power graph of some finite groups are computed.

Consider the dihedral group $D_{2 n}$. In this case $\Gamma_{D_{2 n}}=P_{3}\left[K_{n-1}, K_{1}, \overline{K_{n}}\right]$. If $n$ is a prime power, then $\mathcal{P}\left(D_{2 n}\right)=P_{3}\left[K_{n-1}, K_{1}, \overline{K_{n}}\right]$.

Corollary 2.3. The signless Laplacian eigenvalues of $\Gamma_{D_{2 n}}, n \neq 1$ and $\mathcal{P}\left(D_{2 n}\right)$ where in this case $n$ is a prime power, are $n-2$ with multiplicity $n-2$, 1 with multiplicity $n-1$ and three simple signless Laplacian eigenvalues as follows:

$$
\begin{aligned}
& x_{1}=\frac{\sqrt[3]{-8 n^{3}+45 n^{2}-54 n+27+3 \sqrt{-48 n^{5}+225 n^{4}-420 n^{3}+378 n^{2}-162 n}}}{3} \\
& 3\left(\frac{-4}{9} n^{2}+\frac{2}{3} n-1\right) \\
& -\frac{\sqrt[3]{-8 n^{3}+45 n^{2}-54 n+27+3 \sqrt{-48 n^{5}+225 n^{4}-420 n^{3}+378 n^{2}-162 n}}}{4 n} \\
& +\frac{4 n}{3}-1 \text {, } \\
& x_{2,3}=-\frac{\sqrt[3]{-8 n^{3}+45 n^{2}-54 n+27+3 \sqrt{-48 n^{5}+225 n^{4}-420 n^{3}+378 n^{2}-162 n}}}{6} \\
& +\frac{3\left(\frac{-4}{9} n^{2}+\frac{2}{3} n-1\right)}{2 \sqrt[3]{-8 n^{3}+45 n^{2}-54 n+27+3 \sqrt{-48 n^{5}+225 n^{4}-420 n^{3}+378 n^{2}-162 n}}} \\
& +\frac{4 n}{3}-1 \pm \frac{1}{2} i \sqrt{3} \\
& \left(\frac{\sqrt[3]{-8 n^{3}+45 n^{2}-54 n+27+3 \sqrt{-48 n^{5}+225 n^{4}-420 n^{3}+378 n^{2}-162 n}}}{3}\right. \\
& \left.+\frac{3\left(\frac{-4}{9} n^{2}+\frac{2}{3} n-1\right)}{\sqrt[3]{-8 n^{3}+45 n^{2}-54 n+27+3 \sqrt{-48 n^{5}+225 n^{4}-420 n^{3}+378 n^{2}-162 n}}}\right) .
\end{aligned}
$$


Proof. By Theorem 2.1,

$$
C_{Q}\left(P_{3}\right)=\left(\begin{array}{ccc}
2 n-3 & \sqrt{n-1} & 0 \\
\sqrt{n-1} & 2 n-1 & \sqrt{n} \\
0 & \sqrt{n} & 1
\end{array}\right) .
$$

Now by computing eigenvalues of the matrix $C_{Q}\left(P_{3}\right)$, the result will be completed.

Corollary 2.4. The signless Laplacian eigenvalues of $\Gamma_{T_{4 n}}$ and $\mathcal{P}\left(T_{4 n}\right)$ where in this case $n$ is a power of 2 , are $2 n-2$ with multiplicity $2 n-3,4 n-2$ with multiplicity 1, 2 with multiplicity $n, 4$ with multiplicity $n-1$ and three simple signless Laplacian eigenvalues as follows:

$$
\begin{aligned}
& x_{1}=\frac{2 \sqrt[3]{-8 n^{3}+45 n^{2}-54 n+27+3 \sqrt{-48 n^{5}+225 n^{4}-420 n^{3}+378 n^{2}-162 n}}}{3} \\
& -\frac{3\left(-\frac{16}{9} n^{2}+\frac{8}{3} n-4\right)}{2 \sqrt[3]{-8 n^{3}+45 n^{2}-54 n+27+3 \sqrt{-48 n^{5}+225 n^{4}-420 n^{3}+378 n^{2}-162 n}}} \\
& +\frac{8 n}{3} \text {, } \\
& x_{2,3}=-\frac{\sqrt[3]{-8 n^{3}+45 n^{2}-54 n+27+3 \sqrt{-48 n^{5}+225 n^{4}-420 n^{3}+378 n^{2}-162 n}}}{3} \\
& +\frac{3\left(-\frac{16}{9} n^{2}+\frac{8}{3} n-4\right)}{4 \sqrt[3]{-8 n^{3}+45 n^{2}-54 n+27+3 \sqrt{-48 n^{5}+225 n^{4}-420 n^{3}+378 n^{2}-162 n}}} \\
& +\frac{8 n}{3} \pm \frac{1}{2} i \sqrt{3} \\
& \left(\frac{2 \sqrt[3]{-8 n^{3}+45 n^{2}-54 n+27+3 \sqrt{-48 n^{5}+225 n^{4}-420 n^{3}+378 n^{2}-162 n}}}{3}\right. \\
& \left.+\frac{3\left(-\frac{16}{9} n^{2}+\frac{8}{3} n-4\right)}{2 \sqrt[3]{-8 n^{3}+45 n^{2}-54 n+27+3 \sqrt{-48 n^{5}+225 n^{4}-420 n^{3}+378 n^{2}-162 n}}}\right) .
\end{aligned}
$$

Proof. A simple investigation of dicyclic group $T_{4 n}$ shows that

$$
\Gamma_{T_{4 n}}=W[K_{2 n-2}, K_{2}, \underbrace{K_{2}, K_{2}, \cdots, K_{2}}_{n}],
$$

where $W$ is depicted in Figure 1. If $n$ is a power of 2, then

$$
\mathcal{P}\left(T_{4 n}\right)=W[K_{2 n-2}, K_{2}, \underbrace{K_{2}, K_{2}, \cdots, K_{2}}_{n}] .
$$




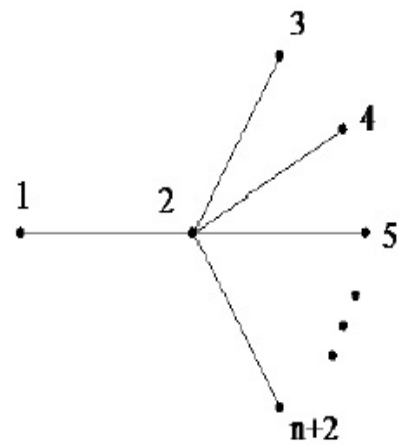

Figure 1. The graph $W$ related to the cyclic graph and power graph of $T_{4 n}$.

So by Theorem 2.1,

$$
C_{Q}(W)=\left(\begin{array}{ccccccc}
4 n-4 & 2 \sqrt{n-1} & 0 & 0 & 0 & \cdots & 0 \\
2 \sqrt{n-1} & 4 n & 2 & 2 & 2 & \cdots & 2 \\
0 & 2 & 4 & 0 & 0 & \cdots & 0 \\
0 & 2 & 0 & 4 & 0 & \cdots & 0 \\
0 & 2 & 0 & 0 & 4 & \cdots & 0 \\
\vdots & \vdots & \vdots & \vdots & \vdots & \ddots & \vdots \\
0 & 2 & 0 & 0 & 0 & \cdots & 4
\end{array}\right) .
$$

Now by computing eigenvalues of the matrix $C_{Q}(W)$, the proof is completed.

Theorem 2.5. (See Tamizh Chelvam and Sattanathan [18]) Let $G$ be an elementary abelian group of order $p^{n}$ for some prime number $p$ and positive integer $n$. Then $\mathcal{P}(G) \cong K_{1}+\cup_{i=1}^{l} K_{p-1}$, where $l=\frac{p^{n}-1}{p-1}$.

Let $E\left(p^{n}\right)$ denote the elementary abelian group of order $p^{n}, p$ is prime, $n$ is positive integer and $l=\frac{p^{n}-1}{p-1}$. By Theorem 2.5, $\mathcal{P}\left(E\left(p^{n}\right)\right) \cong K_{1}+\cup_{i=1}^{l} K_{p-1}$ and so

$$
\mathcal{P}\left(E\left(p^{n}\right)\right) \cong M[K_{1}, \underbrace{K_{p-1}, \cdots, K_{p-1}}_{l}],
$$

where $M=K_{1}+\bar{K}_{l}$.

Corollary 2.6. The signless Laplacian eigenvalues of $E\left(p^{n}\right)$, are $p-2$ with multiplicity $l(p-2), 2 p-3$ with multiplicity $l-1$ and two simple signless Laplacian eigenvalues as follows:

$x_{1,2}=-\frac{3}{2}+\frac{l p}{2}-\frac{l}{2}+p \pm \frac{\sqrt{9+14 l p-10 l-12 p+l^{2} p^{2}-2 l^{2} p-4 l p^{2}+l^{2}+4 p^{2}}}{2}$. 
Proof. By Theorem 2.1 and the structure of $\mathcal{P}\left(E\left(p^{n}\right)\right)$,

$$
C_{Q}(M)=\left(\begin{array}{ccccccc}
l(p-1) & \sqrt{p-1} & \sqrt{p-1} & \sqrt{p-1} & \sqrt{p-1} & \cdots & \sqrt{p-1} \\
\sqrt{p-1} & 2 p-3 & 0 & 0 & 0 & \cdots & 0 \\
\sqrt{p-1} & 0 & 2 p-3 & 0 & 0 & \cdots & 0 \\
\sqrt{p-1} & 0 & 0 & 2 p-3 & 0 & \cdots & 0 \\
\sqrt{p-1} & 0 & 0 & 0 & 2 p-3 & \cdots & 0 \\
\vdots & \vdots & \vdots & \vdots & \vdots & \ddots & \vdots \\
\sqrt{p-1} & 2 & 0 & 0 & 0 & \cdots & 2 p-3
\end{array}\right)
$$

Now by computing eigenvalues of the matrix $C_{Q}(M)$, the result is obtained.

We now state another result of [20] which is crucial in computing normalized Laplacian eigenvalues $\Gamma_{D_{2 n}}, \mathcal{P}\left(D_{2 n}\right), \Gamma_{T_{4 n}}$ and $\mathcal{P}\left(T_{4 n}\right)$.

Theorem 2.7. [20] Let $H$ be a graph with no isolated vertices and $V(H)=$ $\{1,2, \ldots, k\}$, and $G_{i}$ 's be $r_{i}$-regular graphs of order $n_{i}(i=1,2, \ldots, k)$. If $\Gamma=$ $H\left[G_{1}, G_{2}, \cdots, G_{k}\right]$, then normalized Laplacian spectrum can be computed as follows:

$$
\operatorname{Spec}_{\mathcal{L}}(\Gamma)=\left(\bigcup_{i=1}^{k}\left(\frac{N_{i}}{r_{i}+N_{i}}+\frac{r_{i}}{r_{i}+N_{i}}\left(\operatorname{Spec}_{\mathcal{L}}\left(G_{i}\right) \backslash\{0\}\right)\right)\right) \bigcup \operatorname{Spec}\left(\left(C_{\mathcal{L}}(H)\right),\right.
$$

where

$$
N_{i}= \begin{cases}\sum_{j \in N_{H}(i)} n_{j}, & N_{H}(i) \neq \emptyset \\ 0, & \text { otherwise }\end{cases}
$$

and

$$
C_{\mathcal{L}}(H)=\left(c_{i j}\right)_{k \times k}= \begin{cases}\frac{N_{i}}{r_{i}+N_{i}}, & i=j, \\ -\sqrt{\frac{n_{i} n_{j}}{\left(r_{i}+N_{i}\right)\left(r_{j}+N_{j}\right)}}, & i j \in E(H), \\ 0 & \text { otherwise } .\end{cases}
$$

The following results are immediate consequences of Theorem 2.7 and the fact that $\operatorname{Spec}_{\mathcal{L}}\left(K_{n}\right)=\left\{0, \frac{n}{n-1}^{(n-1)}\right\}$.

Corollary 2.8. The normalized Laplacian eigenvalues of $\Gamma_{D_{2 n}}, n \neq 1$ and $\mathcal{P}\left(D_{2 n}\right)$ where in this case $n$ is a prime power, are $\frac{n}{n-1}$ with multiplicity $n-2,1$ with multiplicity $n-1,0$ with multiplicity 1 and two simple normalized Laplacian eigenvalues as follows:

$$
x_{1,2}=\frac{-4 n+1+4 n^{2} \pm \sqrt{32 n^{2}-12 n-28 n^{3}+1+8 n^{4}}}{2\left(2 n^{2}-3 n+1\right)} .
$$


Proof. Consider the dihedral group $D_{2 n}$. In this case $\Gamma_{D_{2 n}}=P_{3}\left[K_{n-1}, K_{1}, \overline{K_{n}}\right]$. If $n$ is a prime power, then $\mathcal{P}\left(D_{2 n}\right)=P_{3}\left[K_{n-1}, K_{1}, \overline{K_{n}}\right]$. By Theorem 2.7,

$$
C_{\mathcal{L}}\left(P_{3}\right)=\left(\begin{array}{ccc}
\frac{1}{n-1} & -\sqrt{\frac{1}{2 n-1}} & 0 \\
-\sqrt{\frac{1}{2 n-1}} & 1 & -\sqrt{\frac{n}{2 n-1}} \\
0 & -\sqrt{\frac{n}{2 n-1}} & 1
\end{array}\right) .
$$

Now by computing eigenvalues of the matrix $C_{\mathcal{L}}\left(P_{3}\right)$, we will prove the result.

Corollary 2.9. The normalized Laplacian eigenvalues of $\Gamma_{T_{4 n}}$ and $\mathcal{P}\left(T_{4 n}\right)$ where in this case $n$ is a power of 2 , are $\frac{2 n}{2 n-1}$ with multiplicity $2 n-3, \frac{4 n}{4 n-1}$ with multiplicity $1, \frac{4}{3}$ with multiplicity $n$, and $\operatorname{Spec}(C)$ that

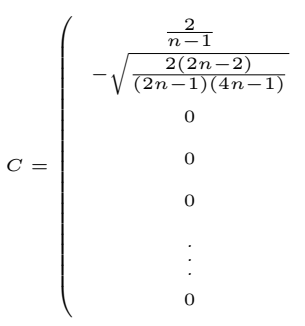

$$
\begin{gathered}
-\sqrt{\frac{2(2 n-2)}{(2 n-1)(4 n-1)}} \\
-\sqrt{\frac{4 n-2}{4 n-1}} \\
-\sqrt{\frac{4}{3(4 n-1)}} \\
-\sqrt{\frac{4}{3(4 n-1)}} \\
-\sqrt{\frac{4}{3(4 n-1)}}
\end{gathered}
$$
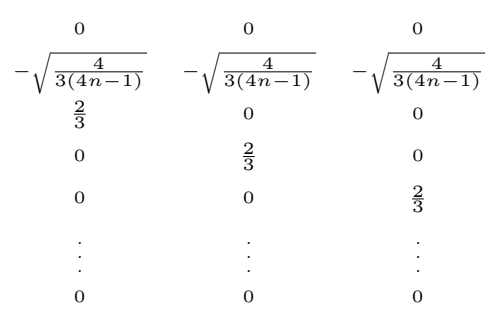

$$
\left.\begin{array}{cl}
\cdots & 0 \\
- & \sqrt{\frac{4}{3(4 n-1)}} \\
\cdots & 0 \\
\cdots & 0 \\
\cdots & 0 \\
\ddots & \vdots \\
\cdots & \frac{2}{3}
\end{array}\right)
$$

Proof. the proof is similar to Corollary 2.8 .

Corollary 2.10. The normalized Laplacian eigenvalues of $E\left(p^{n}\right)$, are $\frac{p}{p-1}$ with multiplicity $l(p-2)+1, \frac{1}{p-1}$ with multiplicity $l-1$ and 0 with multiplicity 1 .

Proof. By Theorem 2.7 and the structure of $\mathcal{P}\left(E\left(p^{n}\right)\right)$,

$$
C_{\mathcal{L}}(M)=\left(\begin{array}{ccccccc}
1 & \sqrt{\frac{1}{l(p-1)}} & -\sqrt{\frac{1}{l(p-1)}} & \sqrt{\frac{1}{l(p-1)}} & \sqrt{\frac{1}{l(p-1)}} & \cdots & \sqrt{\frac{1}{l(p-1)}} \\
\sqrt{\frac{1}{l(p-1)}} & \frac{1}{p-1} & 0 & 0 & 0 & \cdots & 0 \\
\sqrt{\frac{1}{l(p-1)}} & 0 & \frac{1}{p-1} & 0 & 0 & \cdots & 0 \\
\sqrt{\frac{1}{l(p-1)}} & 0 & 0 & \frac{1}{p-1} & 0 & \cdots & 0 \\
\sqrt{\frac{1}{l(p-1)}} & 0 & 0 & 0 & \frac{1}{p-1} & \cdots & 0 \\
\vdots & \vdots & \vdots & \vdots & \vdots & \ddots & \vdots \\
\sqrt{\frac{1}{l(p-1)}} & 2 & 0 & 0 & 0 & \cdots & \frac{1}{p-1}
\end{array}\right) .
$$

Now by computing eigenvalues of this matrix, the proof is completed.

Acknowledgement. This research is supported by National Elites Foundation under Grant Number 15/82041. 


\section{REFERENCES}

[1] Cameron, P.J., and Ghosh, S., The power graph of a finite group, Discrete Math. 311(2011), 1220-1222.

[2] Cameron, P.J., " The power graph of a finite group, II", J. Group Theory 13(2010), $779-783$.

[3] Chattopadhyaya. S., and Panigrahi, P., "On Laplacian spectrum of power graphs of finite cyclic and dihedral groups", Linear Multilinear Algebra, 63:7(2015), 1345-1355.

[4] Hamzeh, A., and Ashrafi, A.R., "The main supergraph of the power graph of a finite group", submitted.

[5] Hamzeh, A., and Ashrafi, A.R., "Automorphism group of supergraphs of the power graph of a finite group", European J. Combin., 60(2017), 82-88.

[6] Hamzeh, A., "Spectrum and L-spectrum of the cyclic group", Southeast Asian Bull. Math., accepted.

[7] Hamzeh, A., and Ashrafi, A.R., "Spectrum and $L$-spectrum of the power graph and its main supergraph for certain finite groups", submitted.

[8] Kelarev, A., Ryan, J., and Yearwood, J., "Cayley graphs as classifiers for data mining: The in uence of asymmetries", Discrete Math., 309:17(2009), 5360-5369.

[9] Kelarev, A.V., and Quinn, S.J., "A combinatorial property and power graphs of semigroups", Comment. Math. Univ. Carolin., 45:1(2004), 1-7.

[10] Kelarev, A.V., Graph Algebras and Automata, Marcel Dekker, New York, 2003.

[11] Kelarev, A.V., and Quinn, S.J., "Directed graphs and combinatorial properties of semigroups", J. Algebra 251:1(2002), 16-26.

[12] Kelarev, A.V., Quinn, S.J., and Smolíková, R., "Power graphs and semigroups of matrices", Bull. Austral. Math. Soc., 63:2(2001), 341-344.

[13] Kelarev, A.V., and Quinn, S.J., "A combinatorial property and power graphs of groups", Contributions to General Algebra, 12 (Vienna, 1999), 229-235, Heyn, Klagenfurt, 2000.

[14] Ma, X.L., Wei, H.Q., and Guo Zhong, "The cyclic graph of a finite group", Algebra, 2013(2013), Article ID 107265, 1-7.

[15] Mehranian, Z., Gholami, A. and Ashrafi, A.R., "The Spectra of power graphs of certain finite groups", Linear Multilinear Algebra, 65:5(2017), 1003-1010.

[16] Rose, J.S., A Course on Group Theory, Cambridge University Prees, Cambridge, New YorkMelbourne, 1978.

[17] Sabidussi, G., "Graph Derivatives", Math. Z., 76(1961), 385-401.

[18] Tamizh Chelvam, T., and Sattanathan, M., "Power graph of finite abelian groups", Algebra Discrete Math. 16:1(2013), 33-41.

[19] West, D.B., Introduction to Graph Theory, Second Edition, Prentice Hall, Inc., Upper Saddle River, NJ, 2001.

[20] Wu, B-F., Lou, Y-Y., and He, C-X., "Signless Laplacian and normalized Laplacian on the H-join operation of graphs", Discrete Math. Algorithm. Appl., 06(2014) [13 pages] DOI: http://dx.doi.org/10.1142/S1793830914500463. 